\title{
Human Centric Data Fusion in Vehicular Cyber-Physical Systems
}

\author{
Aditya Wagh*, Xu Li*, Jingyan $\mathrm{Wan}^{\dagger}$, Chunming Qiao* and Changxu $\mathrm{Wu}^{\dagger}$ \\ * Department of Computer Science and Engineering, State University of New York at Buffalo \\ ${ }^{\dagger}$ Department of Industrial and Systems Engineering, State University of New York at Buffalo
}

\begin{abstract}
Building effective Vehicular Cyber-Physical Systems (VCPS) to improve road safety is a non-trivial challenge, especially when we examine how the driver benefits from the existing and proposed technologies in the presence of Human Factors (HF) related negative factors such as information overload, confusion, and distraction. In this paper, we address a humancentric data fusion problem in VCPS. To the best of our knowledge, this work is the first to apply $\mathrm{HF}$ to the data fusion problem, which has both theoretical value and practical implications. In particular, we present a new architecture by defining a distinct High-Level (HL) data fusion layer with HF considerations, that is placed between the safety applications on the VCPS and the human driver. A data fusion algorithm is proposed to fuse multiple messages (based on reaction time, message type, preferred evasive actions, severity of the hazards, etc) and to maximize the total utility of the messages. The algorithm is tested with real human drivers to demonstrate the potential benefit of incorporating such human-centric fusion in existing warning systems.
\end{abstract}

Index Terms-VCPS, VANET, CPNS, Safety Applications, Data Fusion, Human Factors, ITS, Warning Messages

\section{INTRODUCTION}

Vehicular Cyber-Physical Systems (VCPS) have attracted a significant amount of interest in the past few decades. These networks are also known as Vehicular Ad Hoc Networks (VANET) and offer a platform for a wide range of interesting applications ranging from infotainment, to making the roads safer for motorists and pedestrians. Given their potential, a large amount of research work has been carried out in the areas of routing and data dissemination, aggregation, mobility profiling, and safety applications.

VCPS-based safety applications have typically been designed with a single, clearly defined objective in mind. Most of these applications rely on single-hop communication and their effectiveness is evaluated based on two key aspects: success of delivery and the practical benefit [1]. It is worth noting that VCPS has a human element in the loop since the driver is the final control unit for all generated information. Each of these parameters are critical: we as drivers tend to ignore or disable applications that often raise false alarms. Similarly, warnings that are not delivered within a specific time are useless, if not misleading.

Human Factors (HF) research examines how the driver reacts to hazards and warning notifications. For example, delivering too many messages to the driver at the same time may cause confusion and frustration rather than being helpful. The problem of designing a delivery mechanism that considers the driver's perceptions/reactions is fundamental to the design of effective safety applications and needs to be addressed. However, most of the existing works have not studied this problem looking at both of these aspects at the same time. Our previous work [2] has studied the HF-aware service scheduling problem in VCPS, but the data fusion issue was not addressed.

In this paper, we study the on-board data fusion problem in VCPS from a HF standpoint. First, we propose a new architecture by inserting two distinct fusion layers in to the onboard system. The layer that is placed between the applications and the driver is called the High-Level (HL) Fusion layer and forms the focal point of our work. Next, we design a HL data fusion algorithm that can fuse multiple warning messages sent to the driver by considering their utilities (defined based on HF parameters such as reaction time, message type, driver preference, etc.) and the evasive actions of the driver. Finally, we model an urban scenario using the STISIM driving simulator and test with real drivers to show the potential benefit of the algorithm. It is worth noting that, the on-board data fusion problem is a fundamental issue that will give us a better insight into how drivers repond to warning messages. This will guide us further in optimizing data transmission/fusion in the networking domain. This is not a major concern in this paper due to the limited space and it needs a separate study.

We describe related work in the next section. We introduce our modified multi-layered architecture in Section III and the fusion algorithm in Section IV. Section V discusses the testing scenario and performance metrics for an experiment with human drivers. An analysis of the results are presented in Section VI. We propose areas for future work and conclude our work in the last section.

\section{RELATED WORK}

The idea of fusing data has been applied to VCPS at different levels of operation. For example, we can merge packets to remove redundancy in raw data, i.e. with attributes such as speed, acceleration, etc that are exchanged over the wireless channel. The authors in [3] proposed a component called the Message Dispatcher that acted as a multiplexer at each sender, by removing redundant data elements from application- 
generated messages before sending them on the wireless channel. It demultiplexed and regenerated the messages at the receiver before delivering them to the applications. An allinclusive system that incorporated a Laser-scanner, onboard wireless radio and a Local Dynamic Map was presented in [4]. The fused data from multiple sensors was used to increase the accuracy in sensing the environment. [5] discussed how data fusion could be applied at multiple levels to improve the position, speed and orientation as sensed by the on-board system. The authors in [6] proposed a new Location Division Multiple Access (LDMA) capable of delivering messages across multiple hops in a very short time without causing a broadcast storm. They achieved this by setting up spatial cells and temporal slots using geographical location from a GPS.

A compromise between the traditional layered and unlayered architectures was presented in [7]. The authors proposed an information channel that shared data across layers without going through unnecessary layers. The I-WAY project [8] proposed an architecture capable of inferring hazards and removing unnecessary events and is closely related to our work. However, their process mainly relied on filtration based on predefined rules and merging through the combination of correlated events.

Overall, few existing works have applied HF considerations to the data fusion approach and have studied it entirely from the view point of building an efficient and reliable VCPS.

\section{ARChitecture Design}

The HF-based data fusion algorithm that we propose later in this work is applied to the output of each of the onboard safety applications. To maintain interoperability with the existing safety applications (such as Forward Collision Detection, Speeding Warning, Intersection Violation Detection, etc), we introduce a multi-layered architecture that houses the algorithm in a separate fusion layer. This allows the safety applications to be developed and implemented independently of the fusion process.

The model is placed on top of the existing communication stack as shown in Fig. 1, and fuses data at two distinct stages. Raw data is fused in the Low-Level (LL) fusion layer, which is placed between the applications and the communication stack and sensors. Warning messages such as Speeding, Forward Collusion, and Intersection Violation that are generated by the applications and are directly useful to the human driver are fused in the High-Level (HL) fusion layer, which lies between the applications and the notification system. The notification system may alert the driver using a variety of auditory, visual and tactile modalities.

\section{A. Low-Level Fusion Layer}

The main goal of the LL Fusion layer is to reduce the congestion in the wireless medium. This is crucial from the safety application perspective since most vehicles are constantly in motion, and the range of the on-board radio is only a few hundred feet, thereby giving us a very small time-window

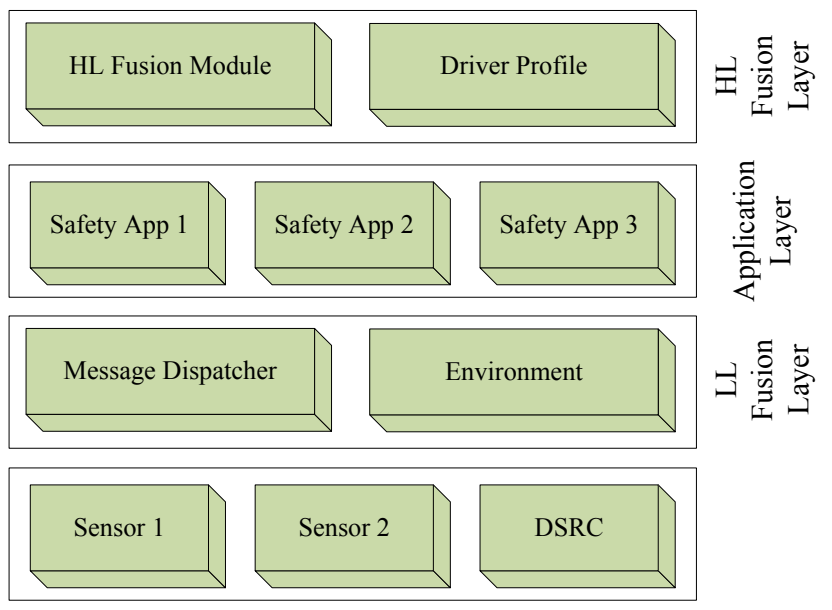

Fig. 1: Modified Multi-Layered Architecture for Data-Fusion

for communication. We have presently used the Message Dispatcher [3] to handle this task. The LL Fusion layer also performs two additional functions: it checks incoming data for correctness and maintains a historical information repository called the environment.

\section{B. High-Level Fusion Layer}

HL fusion forms the basis for our work and is placed between the applications and the driver. It is directly responsible for applying HF considerations to the application layer output, rendering it more useful to the driver. In particular, the HL fusion algorithm uses the driver's decisions to determine the redundancy and importance of individual warnings that are queued for notification. It queries and uses information from the Driver Profile module to customize the Warning Messages to the driver. The details of the process are discussed in section IV-B.

The Driver Profile module supplies the fusion algorithm with the necessary data via lookup functions. This includes the driver's preferences as well as the quality of the driver's responses to warning messages. For example, an elderly driver might prefer to hear a message using the audio system in addition to a tactile stimulus. They might also wish to have messages repeated if no response is detected within a certain time.

\section{High-LeVel Data Fusion}

In order to fuse various warning messages appropriately, we need to carefully examine the driver's familiarity and habitual response to each message. For example, two drivers might react very differently to the same message and thus it is important to use personalized information during fusion. Once this is done, we must schedule the messages based on how useful the driver finds each message if it is delivered at a specific time using a specific modality. We refer to the total benefit that results from the notification as the overall utility of that message. 
The nature of the reaction and the reaction time also both depend on a number of other factors such as the the frequency of the message, the modality on which it is delivered, the location of the hazard relative to the vehicle and the time elapsed since the last warning message. The algorithm combines them using a utility function that is subsequently used to compare messages when there is a conflict. We discuss the message type utility first since it is used extensively in the data fusion algorithm and later outline the other types of utilities that are used in computing the overall benefit.

\section{A. Implications of the Message Type}

The authors in [9] showed that the repetition of a stimulusreponse pair leads to successively better response times. In other words, if a driver has responded to a particular stimulus, such as a specific hazard, multiple times in the past, it is more strongly linked in their long term memory. Thus, when choosing between two kinds of warning messages that require the same evasive actions from the driver, it would be preferable to notify them of the more familiar hazard. Further, the response to repeated events is better than when different kinds of events are alternated.

For a particular message in the queue, we denote its typebased utility based on three factors, $N$, the total number of messages in the queue, $n$, the number of messages of its own type that follow it and $f ; 0<f \leq 1$, the driver's familiarity with this type of message:

$$
U_{\text {type }}=f \times n / N
$$

We note that there are limits on the usefulness of repetitions and that increasing the frequency beyond a certain limit, depending on the message, does not improve the response from an individual [10].

Hence, once $f$ reaches a certain threshold, we simply denote the utilty as:

$$
U_{\text {type }}=n / N
$$

\section{B. High Level Fusion Algorithm}

In most emergency situations, a number of correlated hazards are detected simultaneously, resulting in a large number of messages being sent to the notification system. According to the Hick-Hyman law [11], the reaction time increases linearly with the stimulus information. Bombarding the driver with excessive amounts of information can confuse them and increase reaction time. Furthermore, a short time interval (less than $100 \mathrm{~ms}$ ) between two subsequent notifications can lead to responses to both closely placed notifications getting delayed and appearing together. This interval is also called the Stimulus Onset Asynchrony (SOA).

The HL Fusion algorithm solves this problem by combining multiple correlated warnings that are generated by the underlying safety applications, from the HF standpoint, thereby attempting to pass on only the most critical information to the driver.
TABLE I: Additional information for hazards $H_{1}$ through $H_{5}$

\begin{tabular}{|c|c|l|}
\hline Hazard & Location & Action Set \\
\hline$H_{1}$ & $L_{1}$ & $\left(L_{1}, D_{1}, S_{1}\right),\left(L_{2}, D_{2}, S_{2}\right)$ \\
\hline$H_{2}$ & $L_{1}$ & $\left(L_{1}, D_{1}, S_{2}\right)$ \\
\hline$H_{3}$ & $L_{3}$ & $\left(L_{1}, D_{1}, S_{1}\right),\left(L_{2}, D_{2}, S_{2}\right)$ \\
\hline$H_{4}$ & $L_{4}$ & $\left(L_{4}, D_{4}, S_{4}\right)$ \\
\hline$H_{5}$ & $L_{5}$ & $\left(L_{4}, D_{4}, S_{4}\right),\left(L_{5}, D_{5}, S_{5}\right)$ \\
\hline
\end{tabular}

The algorithm handles evasive maneuvers by quantizing and encoding three key parameters emerging from each maneuver: the lane choice, the direction and the speed. With the help of this kind of encoding, each action can be described as a triplet of type (Lane Choice, Direction, Speed).

We generate the set of evasive actions for the entire set of warning messages by querying the driver profile module which provides a lookup function for each warning message. The key idea is to pick a small subset of this set while ensuring that the subset carries all the information necessary to successfully avoid all the hazards. This is accomplished through a three step reduction based on hazard location and the set of evasive actions for different messages. Finally, we find modalities and notification delays for each of the warning messages in the subset such that we get a maximum value of the overall utility.

In order to understand the algorithm better, we present the following example consisting of 5 hazards, namely $H_{1}$ through $H_{5}$. The encoded action set triplets and additional information for these hazards are listed in Table I.

In the absence of the fusion algorithm, the system generates and displays warnings $W_{1}$ through $W_{5}$ corresponding to the hazards. To prevent this, we remove redundancy through a three step process as follows:

Step 1. Location-based: $H_{1}$ and $H_{2}$ appear to be originating at the same location. We find the utility $U_{\text {type }}$ of the corresponding warnings $W_{1}$ and $W_{2}$ and choose the one (say $H_{1}$ ) that offers higher benefit.

Step 2. Matching Actions: From the remaining hazards, we look for those pairs that require the same actions from the driver. We identify $H_{1}$ and $H_{3}$ and find the value of $U_{\text {type }}$ for each. Let us assume that $H_{3}$ has a higher value, and so we discard $H_{1}$.

Step 3. Action Subsets: Finally, we identify hazards whose corresponding action sets form subsets of other hazards. This leads us to $H_{4}$ and $H_{5}$. In such a case, we choose the one with the smaller action set since any evasive maneuver chosen from it is an equally valid response for the other hazard; here we choose $\mathrm{H}_{4}$.

We have just reduced our hazards from 5 down to 2, namely $H_{3}$ and $H_{4}$. This means that we would only need to display warnings $W_{3}$ and $W_{4}$ that correspond to them. At this stage, we need to set up the notification schedule for the two warnings by picking the correct modality and time for diplay. This is done by maximizing the overall utility function $U_{\text {total }}$ given by Eq. (6) from section IV-C. This is an NPhard problem that can be solved using a number of heuristic approaches. We do not dicuss and compare different heuristic approaches here since the discussion warrants a study that is 
independent of the fusion algorithm.

The utility function $U_{\text {total }}$ maps the parameter space of the 4-tuples (Warning Message Type, Hazard Location, Chosen Modality, Notification Delay) onto the set of Real numbers. Here, the delay refers to the time after which the warning will be presented on that modality. The values returned by the function can be used to compare different 4-tuples.

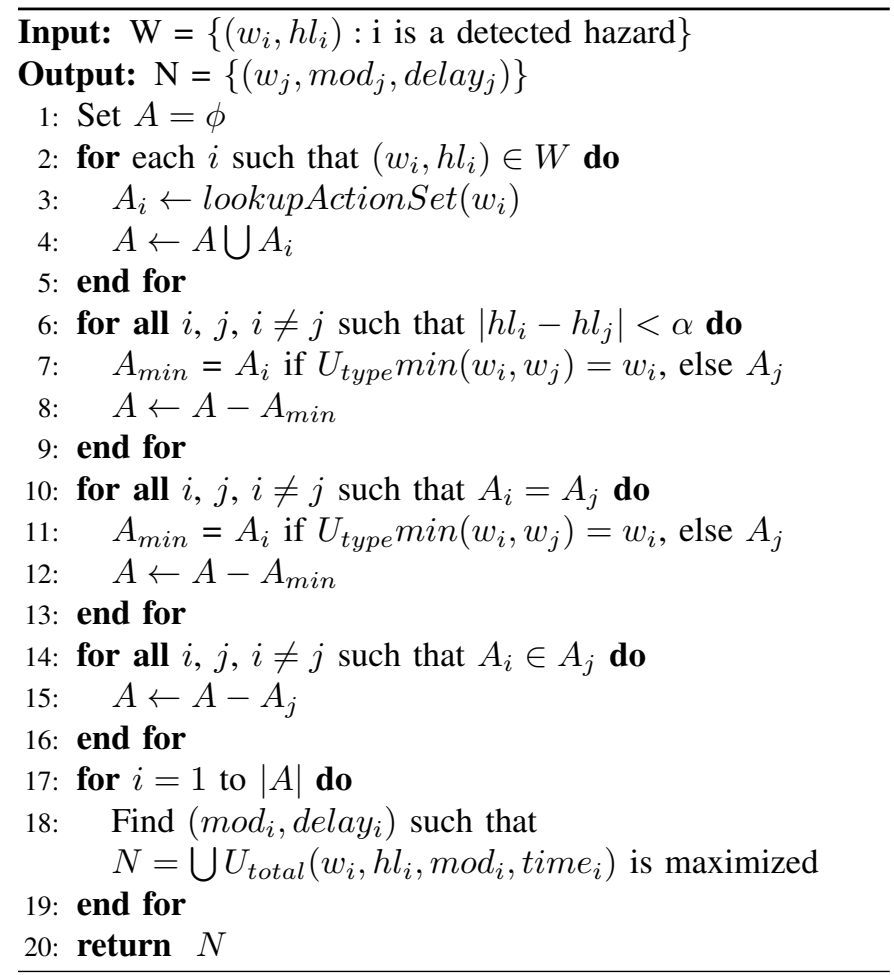

Fig. 2: Formal description of HL Fusion Algorithm

A formal description of the algorithm is presented in Fig. 2 , where the input is a set of pairs of warning messages and hazard locations represented by $w$ and $h l$ respectively, and the output is the set of triplets with modality and notification times for each warning message.

\section{Computing the Overall Utility}

We include three additional aspects from HF in computing the overall utility of the messages:

1) Hazard Location: The driver's cognitive processes under time pressure such as the need to react immediately causes errors. We assign higher utility to messages when they are delivered at sufficient distances from the hazard. The distance or notification range (NR) that is associated with each type of message is stored in the driver profile module. Thus, we define the utility based on the hazard location as:

$$
U_{l o c}= \begin{cases}D / V & \text { within NR } \\ (D / V) \times \alpha_{\text {loss }} & \text { otherwise }\end{cases}
$$

where $D$ is the distance from the vehicle to the hazard, $V$ is the velocity of the vehicle, and $\alpha_{\text {loss }} ; 0 \leq \alpha_{\text {loss }}<1$, is a constant used to suppress the utility when the hazard lies outside the NR.
2) Choice of Notification Modalities: It has been shown that people respond better to succesive messages when they are delivered over different modalities than a single one [12]. Another important issue is the difference in reaction times for different modalities [13].

We use $t_{v i s}$ to denote the time that a driver has, to react to a visual message before being notified about the next visual message and $t_{\text {aud }}$ for the time for a corresponding time frame between two auditory messages. We also include $\beta$, that increases with the number of times the driver has heard the message and $P$, that represents the driver's proficiency at reading the visual message

$$
U_{\text {mod }}= \begin{cases}1 /\left(t_{\text {aud }}-\beta\right) & \text { auditory modality } \\ P \times 1 / t_{v i s} & \text { visual modality }\end{cases}
$$

3) Notification Time: The notification time has two important consequences on the driver's response: firstly, the time that is available to understand and to react to the message determines the effectiveness of the action performed. Secondly, messages must be spaced out in the temporal domain to avoid short SOA. This is based on the observation that the human being can only focus on a single task in terms of decision making and response selection [14].

If we denote the reaction time for the previous message as $T_{\text {prev }}$, and the time between the end of the previous message and the beginning of the current message as $G$, we may express the utility for the notification delay as:

$$
U_{\text {time }}= \begin{cases}1 & \text { first message or } G \geq T_{\text {prev }} \\ G / T_{\text {prev }} & G<T_{\text {prev }}\end{cases}
$$

Based on this discussion, we can now calculate the overall utility for a message by combining the four aspects:

$$
U_{\text {total }}=U_{\text {type }} \times U_{\text {loc }} \times U_{\text {mod }} \times U_{\text {time }}
$$

\section{EXPERIMENT DESIGN}

We set up an HF-oriented experiment to study the potential benefit of our algorithm using the STISIM driving simulator with real drivers. We chose an intersection violation event for testing the algorithm. This scenario involved 5 distinct warnings as shown in table II.

We tested the response to violating cross traffic in the following three situations where the subject vehicle was equipped with:

1) No warning system

2) A warning system without data fusion

3) A warning system with data fusion

Test drivers drove a vehicle in an urban environment, along a straight, four-lane road (two lanes in either direction), that was 89,000 feet long. They encountered a total of 29 intersections along the way with the violating cross traffic vehicles at 9 of them. We programmed a sedan and a truck as lead vehicles for the subject. The road was lined with buildings on either side, 
TABLE II: List of Warnings used in Simulation

\begin{tabular}{|l|c|c|l|}
\hline Warning & Abbr. & Source & Trigger \\
\hline $\begin{array}{l}\text { Forward } \\
\text { Collision Warning }\end{array}$ & FCW & Local & $\begin{array}{l}\text { Obstacle detected directly in } \\
\text { path of the subject vehicle }\end{array}$ \\
\hline $\begin{array}{l}\text { Hard Braking } \\
\text { Warning }\end{array}$ & HBW & Radio & $\begin{array}{l}\text { Large deceleration detected } \\
\text { nearby }\end{array}$ \\
\hline $\begin{array}{l}\text { Intersection } \\
\text { Violation Warning }\end{array}$ & IVW & Radio & $\begin{array}{l}\text { Nearby vehicle ran red light } \\
\text { or broke an intersection rule }\end{array}$ \\
\hline Speeding Warning & - & Local & $\begin{array}{l}\text { Subject vehicle has crossed } \\
\text { posted speed limit }\end{array}$ \\
\hline Overtaking Warning & - & Radio & $\begin{array}{l}\text { Vehicle overtaking from the } \\
\text { subject vehicle's rear }\end{array}$ \\
\hline
\end{tabular}

making it difficult to spot the cross traffic until it had driven into the intersection. At this point, the lead vehicles would panic and hit the brakes, swerving and coming to a stop at random points around the intersection. Fig. 3 shows such an intersection just before the two lead vehicles respond to the violating cross traffic.

The scenario was set up on a computer running the STISIM driving simulator. The test drivers interacted with the simulator using a steering wheel, and gas and brake pedals. The subject vehicle was equipped with automatic transmission and was allowed to reach speeds up to $70 \mathrm{mph}$. The drivers were subjected to realistic audio feedback from the simulator and were informed about the various warnings.

The Speeding Warning was delivered when the drivers exceeded a speed of $40 \mathrm{mph}$. The HBW was received via the wireless channel from other vehicles whereas the FCW was generated locally based on radar and camera sensing. All warnings were delivered over the audio and video interface. From the subjects perspective, an IVW, two lead vehicle HBWs, multiple FCWs and a Speeding Warning were received. The first violation warning notification started at 400 feet from the intersection and the warnings were spread with a $200-400 \mathrm{~ms}$. gap between the end of one audio notification and the start of the next.

Without Fusion, the notification system attempted to deliver as many warnings as it could on a first-come first-served basis.

Using the high level fusion algorithm, we needed to warn the subject only about the FCW since the actions induced by it also cover the HBW and IVW warnings. Thus, in this case, the system repeated the FCW at regular intervals near the intersection.

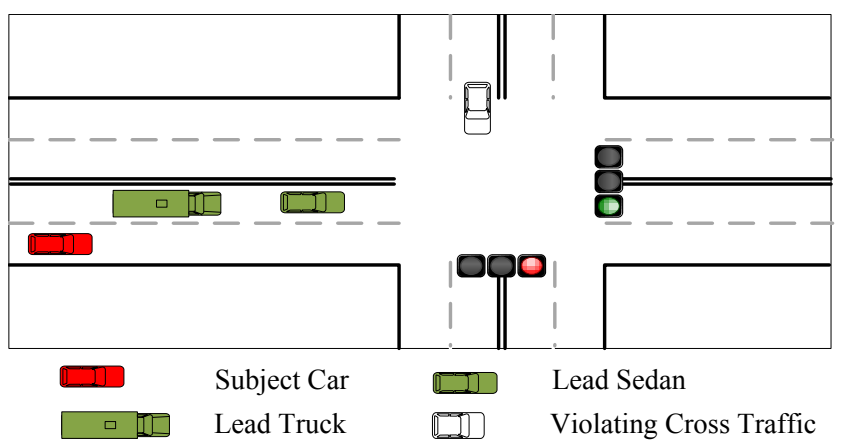

Fig. 3: An intersection with Violating Cross Traffic

\section{Vi. Performance Analysis}

We initially noticed that the drivers started relying heavily on the system from the time that the first warning was delivered. Most drivers traveled without slowing down through all subsequent intersections when no warnings were provided.

We present the observed probabilities for different driver responses at intersections with violating cross traffic, across the Fusion, No Fusion and No Warning cases in Fig. 4. These were classified as No Deceleration, if the driver failed to decelerate through the enitre intersection, Slow Decelration, if the driver decelerated but could not have stopped behind the lead vehicles and Fast Deceleration, if the driver had slowed sufficiently to be able to stop behind the lead vehicles. In quantitative terms, deceleration values ranging between $(0,-11] \mathrm{ft} / \mathrm{sec}^{2}$ were classified as slow deceleration and between $[-17,-20.91] \mathrm{ft} / \mathrm{sec}^{2}$ as fast deceleration. The fast deceleration was bounded by the limit enforced by STISIM. The simulator also simulated realistic braking distances in addition to sounds and vehicle control for these braking values. With fast deceleration, the driver could stop at least $30 \mathrm{ft}$ from the closest lead vehicle, and $100 \mathrm{ft}$ from the intersection if they applied the brakes fully.

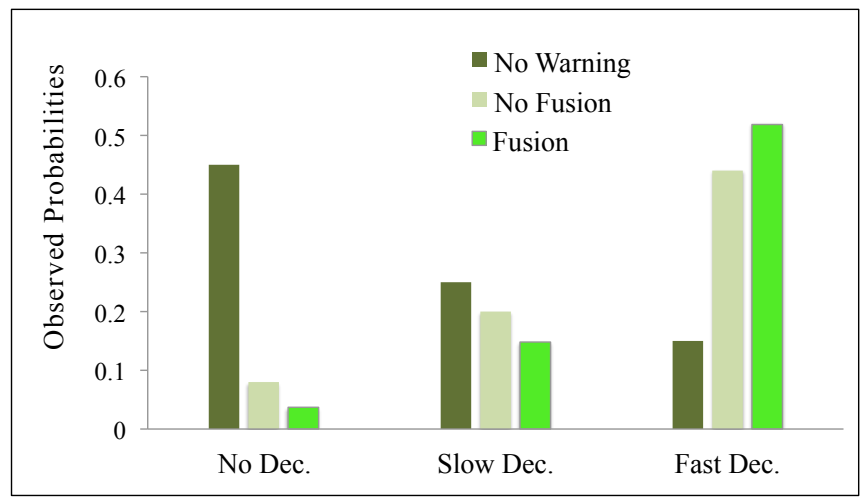

Fig. 4: Observed probabilities for No Deceleration, Slow Deceleration, and Fast Deceleration responses at intersections with violating cross traffic in Fusion, No Fusion and No Warning scenarios

Comparing the reactions at intersections where the notification system was disabled, with the intersections where the warnings were delivered, we saw that most drivers in the former case failed to apply the brakes in time. Here, the dominant reaction was to swerve across obstacles without decelerating. This can be seen by the sharp increase in the No Deceleration response.

We are more interested in comparing the driver's response to the output of the fusion algorithm as compared with the unfiltered output from all the safety applications and here too we see a difference in the reactions. When presented with only a single warning (FCW), most drivers chose to slow down immediately. Although a few drivers chose to ignore the warnings completely, it was rare for most to continue at full speed given the warnings. There were improvements across 
all the parameters in the presence of data fusion. Comparing the fusion and non-fused warnings systems, the chances that the driver would not decelerate at all were cut down by $50 \%$. Instances of delayed decelerations were reduced by $20 \%$, while fast decelerations went up by $27 \%$. Note that these figures may be much less dramatic in a more realistic scenario but they certainly show hope for improving the existing system. Every little increase in the efficiency of the safety system could potentially save another human life.

We also included two other events: Stopped, when the driver stopped behind the lead vehicles and Slow reaction, when the driver took at least 2 seconds to respond after the first audiovisual warning notification, either though braking or changing their direction. These are shown in Fig. 5.

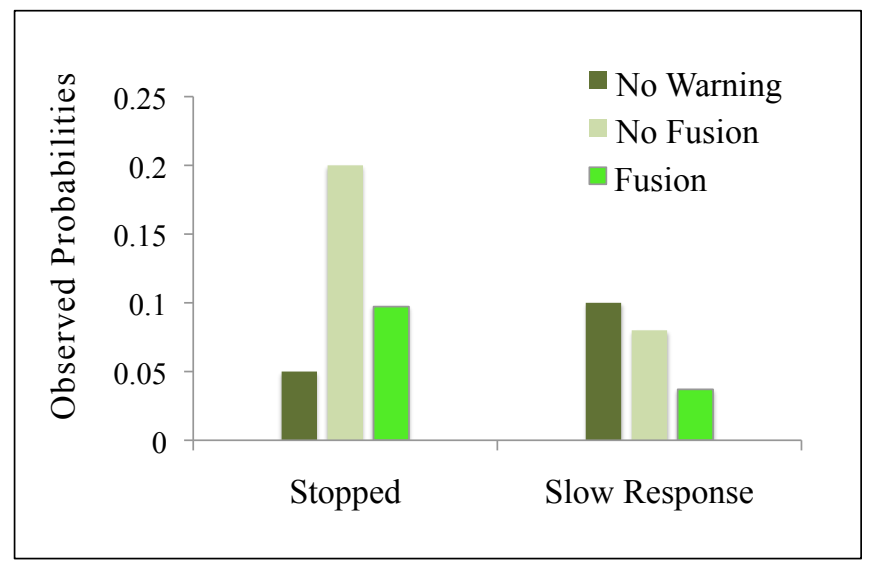

Fig. 5: Observed probabilities for stopping and slow response times at intersections with violating cross traffic in Fusion, No Fusion and No Warning scenarios

Here, it is important to note that coming to a complete stop is not necessarily the best course of action. Most drivers decelerated rapidly when provided with the fused warnings and thus were able to choose between stopping and driving around the lead vehicles if it was safe to do so. In the nonfused scenario, we saw the the drivers had applied brakes slower and this caused some of them to attempt to bring the vehicle to a stop rather than going around the lead vehicles. As expected, drivers with no warnings performed poorly across all the observed parameters.

We also saw that the chance of a driver responding slower to the hazard was much lower using high level fusion as compared with the other two scenarios. Overall, most drivers instinctively slowed down regardless of the warning type. In the fusion case, they could react better since there was less information to process and it could consequently be used more efficiently to make better decisions.

\section{CONCLUSION}

The architecture and the algorithm presented in this paper shows noticeable improvements over a non-intelligent notification system and it can be improved even further by calibrating the Driver Profile in real time based on the drivers responses to the warnings. We plan to incorporate utility-based routing into the LL fusion layer to ease the congestion on the wireless channel. Again, the utility of particular data elements would be computed by considering the usefulness to a human driver at that particular time. The fusion algorithm cannot merge certain correlated warnings because their action sets are mismatched. In such cases, we wish to present a modified notification rather than two separate notifications.

We proposed a fusion algorithm to reduce the amount of non-beneficial information that was delivered to the driver and designed a layered architecture for incorporating this in the on-board warning system. We observed that subjects reacted better to hazards when they were given fewer more relevant warnings as compared with a large number of correlated warnings. We believe that our work can be incorporated into other areas such as notification systems for soldiers, and modified for corresponding aviation and naval systems.

\section{ACKNOWLEDGMENTS}

We are grateful to Yi Tang and Xuegang Yu for their contribution to this work. This research is partially supported by National Science Foundation of USA Grant NSF-CPS1035733 and the Cisco University Research Program.

\section{REFERENCES}

[1] J. Yin, T. ElBatt, G. Yeung, B. Ryu, S. Habermas, H. Krishnan, and T. Talty, "Performance evaluation of safety applications over dsrc vehicular ad hoc networks," in Proceedings of the 1st ACM international workshop on Vehicular ad hoc networks. ACM, 2004, pp. 1-9.

[2] X. Li, X. Yu, A. Wagh, and C. Qiao, "Human factors-aware service scheduling in vehicular cyber-physical systems," in INFOCOM, 2011.

[3] C. L. Robinson and L. C. D. Caveney, "Efficient coordination and transmission of data for cooperative vehicular safety applications," in in the Proc. of the 3rd international, 2006, pp. 10-19.

[4] F. Ahlers and C. Stimming, "Cooperative Laserscanner Pre-DataFusion,” in Intelligent Vehicles Symposium, 2008, pp. 1187-1190.

[5] A. Boukerche, H. A. B. F. Oliveira, E. F. Nakamura, and A. A. F Loureiro, "Vehicular Ad Hoc Networks: A New Challenge for Localization-Based Systems," Comput. Commun., vol. 31, no. 12, pp. 2838-2849, July 2008.

[6] R. Mangharam, R. Rajkumar, M. Hamilton, P. Mudalige, and F. Bai, "Bounded-latency alerts in vehicular networks," in 2007 Mobile Networking for Vehicular Environments, May 2007, pp. 55-60.

[7] H. Fler, M. Torrent-moreno, M. Transier, A. Festag, and H. Hartenstein, "Thoughts on a protocol architecture for vehicular ad-hoc networks," in 2nd Int. Workshop on Intelligent Transportation (WIT), 2005.

[8] G. Rigas, P. Bougia, D. Fotiadis, C. Katsis, and A. Koutlas, "Iway: Towards highway vehicle-2-vehicle communication and driver support," in Systems, Man and Cybernetics, 2008. SMC 2008. IEEE International Conference on, Oct. 2008, pp. 3376-3381.

[9] S. Kornblum, "Sequential effects in choice reaction time. a tutorial review," Attention and Performance IV, In Kornblum (Ed.), 1973.

[10] W. Rogers, G. Rousseau, and A. Fisk, "Automatic and controlled processing theory and its applications to human factors problems," Handbook of Applied Cognition, In F.T. Durso (Ed.), pp. 33-56, 1999.

[11] W. Hick, "On the rate of gain of information," Quaterly Journal of Experimental Psychology, 4, pp. 11-26, 1952.

[12] C. Wickens, D. Sandry, and M. Vidulich, "Compatibility and resource competition between modalities or input, output, and central processing," Human Factors, vol. 25, pp. 227-248, 1983.

[13] F. Galton, "On instruments for (1) testing perception of differences of tint and for (2) determining reaction time," Journal of the Anthropological Institute, vol. 19, pp. 27-29, 1889.

[14] H. Pashler, The Psychology of Attention. MIT Press, MA, 1998. 